MATHEMATICS OF COMPUTATION

Volume 72, Number 244, Pages 1655-1673

S 0025-5718(03)01473-X

Article electronically published on April 28, 2003

\title{
STABILIZED HYBRID FINITE ELEMENT METHODS BASED ON THE COMBINATION OF SADDLE POINT PRINCIPLES OF ELASTICITY PROBLEMS
}

\author{
TIANXIAO ZHOU
}

\begin{abstract}
How, in a discretized model, to utilize the duality and complementarity of two saddle point variational principles is considered in the paper. A homology family of optimality conditions, different from the conventional saddle point conditions of the domain-decomposed Hellinger-Reissner principle, is derived to enhance stability of hybrid finite element schemes. Based on this, a stabilized hybrid method is presented by associating element-interior displacement with an element-boundary one in a nonconforming manner. In addition, energy compatibility of strain-enriched displacements with respect to stress terms is introduced to circumvent Poisson-locking.
\end{abstract}

\section{INTRODUCTION}

Due to the good performances of several 4-node quadrilateral elements [9]-[14] recently, there is a renewed interest in the construction of finite element schemes that enhance or enrich standard displacement schemes. The two aims of this enhancement are (1) to achieve a greater order of accuracy when using coarse meshes, and (2) to circumvent the locking response in the nearly incompressible regime while preserving the convenience of the displacement finite element methods. The different approaches, such as the assumed stress hybrid method 90 10] and the enhanced strain method 11-14 have been considered in the literature. Rational use of accuracy-enhanced displacements of nonconforming bubbles type is fundamental to these methods. But, up to now, there is no mathematical conclusion for any good numerical performance of these lower order elements. In particular, the condition that guarantees the achievement of higher accuracy and the circumvention of the locking response is not known. Besides in the past, two classes of saddle point problems, derived from the potential energy principle and the complementary energy principle, respectively, were discretized each in isolation, and the duality and complementarity of mechanical variational principles was not taken into account for the construction of discretized models of high performance. Then the so-called inf-sup condition [1] makes optimal construction complicated.

Following the basic idea of [18], we consider how to incorporate the strong points of two mutually dual aspects into a hybrid model. The so-called third-class hybrid variational method, different from the conventional hybrid methods, is given. The

Received by the editor October 13, 1999 and, in revised form, March 7, 2001.

2000 Mathematics Subject Classification. Primary 65N12, 65N30.

This work was subsidized by the Special Funds for Major State Basic Research Projects (G1999032801) and the Funds for Aeronautics (00B31005). 
new method is based on a linear combination of two dual systems of saddle point conditions. One of the two saddle point problems used is the domain-decomposed Hellinger-Reissner principle, another is a dual to the former, primal hybrid variational principle. The paper shows that this combination leads to a homology family of optimality conditions for the two variational principles, and the new hybrid finite element method does not require the inf-sup condition. Thus the method is stabilized, but different from the various least square methods 3, 4, 5, 8. For convenience, it will be referred to as the combined hybrid method.

In addition, based on the convergence analysis for the stabilized hybrid method, a concept of generalized compatibility, the so-called index of energy compatibility is introduced to answer the previously mentioned question. It is pointed out in this paper that the index of a nonconforming displacement subspace with respect to a given stress space is crucially important to construct the "good" lower-order elements.

The layout of the paper is as follows. The second section is devoted to derivation of the homology family of optimality conditions for the saddle point variational principles of elasticity. Then a mathematical foundation of the stabilized hybrid method is established in the third section, and the convergence error estimates that introduce the concept of index of energy compatibility are derived. In the final section, the index of energy compatibility (E-C index) is defined. The higher E-C index of the Wilson displacement mode with respect to the Pian-Sumihara stress mode [9] is verified. The effectiveness of the E-C index on improvement of accuracy and stability is illustrated by two new quadrilateral elements of lower order.

\section{Combined VARIATIONAL PRINCIPLE}

Let us consider the following elasticity problems:

$$
\left.\begin{array}{rlrl}
-\operatorname{div} \sigma & =f \\
\sigma & =D[\varepsilon(u)] \\
\varepsilon(u) & =\frac{1}{2}\left(\nabla u+\nabla^{T} u\right)
\end{array}\right\} \begin{aligned}
& \\
& u=0 \text { on } \partial \Omega,
\end{aligned}
$$

where $u$ is the displacement field, $\sigma$ the stress tensor, $\varepsilon$ the strain, $f$ the body force, $D$ the fourth-order tensor of the elastic moduli, $\Omega$ the region in $R^{n}(n=2,3)$ with boundary $\partial \Omega$.

Besides the usual symmetry properties for $D$, assume that there exists a constant $C>0$ such that for any $\varepsilon(v)$,

$$
D[\varepsilon(v)] \cdot \varepsilon(v) \geq C \varepsilon(v) \cdot \varepsilon(v) .
$$

In the paper, the Sobolev spaces $H^{m}(\Omega), H_{0}^{m}(\Omega)$ and other relative notation such as the divergence space $H(\operatorname{div}, \Omega)$, norm $\|\cdot\|_{m, n}$, semi-norm $|\cdot|_{m}$ and so on, will be employed without explanation. For the meaning of these notations, see [1].

It is known that the principles of minimum potential energy and minimum complementary energy are two basic variational approaches to a solution of the problem, and the displacement space $\left(H_{0}^{1}(\Omega)\right)^{n}$ and the stress spaces $H(\operatorname{div} ; \Omega):=$ $\left\{\tau \in\left(L^{2}(\Omega)\right)^{\frac{n(n+1)}{2}} \mid: \operatorname{div} \tau \in\left(L^{2}(\Omega)\right)^{n}\right\}$ are two basic solution spaces. 
For the finite element needs of relaxing continuity, some piecewise Sobolev spaces are introduced in various mixed/hybrid methods to replace $\left(H_{0}^{1}(\Omega)\right)^{n}$ or/and $H(\operatorname{div}, \Omega)$. In the paper, the following spaces are considered:

$$
\begin{gathered}
\mathbf{V}=\prod_{\Omega_{i} \in \mathrm{T}_{h}} H\left(\operatorname{div}, \Omega_{i}\right), \\
\mathbf{U}=\left\{v \in\left(\prod_{\Omega_{i} \in \mathrm{T}_{h}} H^{1}\left(\Omega_{i}\right)\right)^{n}|: v|_{\partial \Omega}=0\right\},
\end{gathered}
$$

where $\mathrm{T}_{h}=\left\{\Omega_{i}\right\}$ denotes a (finite element) subdivision of $\Omega$. Because both the displacement continuity and the normal stress continuity across the interfaces $\partial \Omega_{i}$ are relaxed simultaneously, we yet need a Lagrange multiplier space, i.e., the displacement trace space

$$
\mathbf{U}_{c}=\left(\frac{H_{0}^{1}(\Omega)}{\prod_{i} H_{0}^{1}\left(\Omega_{i}\right)}\right)^{n}=\left\{\text { trace of } v \in\left(H_{0}^{1}(\Omega)\right)^{n} \text { at } \prod_{i} \partial \Omega_{i}\right\} .
$$

For the stress/displacement space $\mathbf{V} \times\left(\mathbf{U} \times \mathbf{U}_{c}\right)$, there are also two variational approaches to the solution of problem (2.1). In other words, we have the following theorem.

Theorem 2.1. Assume that $f \in\left(L^{2}(\Omega)\right)^{n}$. The problem (2.1) is equivalent to any one of the following two saddle point problems.

Find $\left(\sigma, u, u_{c}\right) \in \mathbf{V} \times \mathbf{U} \times \mathbf{U}_{c}$ such that

$$
\begin{array}{ll}
\mathbf{a}(\sigma, \tau)-\mathbf{b}_{2}(\tau, u)+\mathbf{b}_{1}\left(\tau, u-u_{c}\right)=0 & \forall \tau \in \mathbf{V}, \\
\mathbf{b}_{2}(\sigma, v)-\mathbf{b}_{1}\left(\sigma, v-v_{c}\right)=(f, v) & \forall\left(v, v_{c}\right) \in \mathbf{U} \times \mathbf{U}_{c} .
\end{array}
$$

Find $\left(\sigma, u, u_{c}\right) \in \mathbf{V} \times \mathbf{U} \times \mathbf{U}_{c}$ such that

$$
\begin{aligned}
-\mathbf{b}_{1}\left(\sigma, v-v_{c}\right)+\mathbf{d}(u, v) & =(f, v) & & \forall\left(v, v_{c}\right) \in \mathbf{U} \times \mathbf{U}_{c}, \\
\mathbf{b}_{1}\left(\tau, u-u_{c}\right) & =0 & & \forall \tau \in \mathbf{V},
\end{aligned}
$$

where

$$
\begin{aligned}
\mathbf{a}(\sigma, \tau) & =\int_{\Omega} \sigma \cdot D^{-1}[\tau] d \Omega, & \mathbf{d}(u, v) & =\sum_{\Omega_{i} \in \mathrm{T}_{h}} \int_{\Omega_{i}} \varepsilon(u) \cdot D[\varepsilon(v)] d \Omega, \\
\mathbf{b}_{1}(\tau, v) & =\sum_{\Omega_{i} \in \mathrm{T}_{h}} \oint_{\partial \Omega_{i}}(\tau \cdot \vec{n}) \cdot v d s, & \mathbf{b}_{2}(\tau, v) & =\sum_{\Omega_{i} \in \mathrm{T}_{h}} \int_{\Omega_{i}} \tau \cdot \varepsilon(v) d \Omega,
\end{aligned}
$$

and $\vec{n}$ denotes the unit outer normal to $\partial \Omega_{i}$.

Proof. By virtue of the integration by parts formula

$$
\int_{K} \sigma: \varepsilon(v) d \Omega=\oint_{\partial K}(\sigma \cdot \vec{n}) \cdot v d s-\int_{K} \operatorname{div} \sigma \cdot v d \Omega,
$$

the problem 2.1 can be changed into the weak formulation

$$
\begin{array}{ll}
\sum_{i}\left[\int_{\Omega_{i}} \tau \cdot D^{-1} \sigma d \Omega-\int_{\Omega_{i}} \tau \cdot \varepsilon(u) d \Omega\right]=0 & \forall \tau \in \mathbf{V}, \\
\sum_{i}\left[\int_{\Omega_{i}} \sigma \cdot \varepsilon(v) d \Omega-\oint_{\partial \Omega_{i}}(\sigma \cdot \vec{n}) \cdot v d s\right]=(f, v) & \forall v \in \mathbf{U} .
\end{array}
$$


Setting $u_{c}=\left.u\right|_{\partial \Omega_{i}}$ and recalling that $\sigma=D \varepsilon(u) \in H(\operatorname{div} ; \Omega)$ and for $v_{c} \in$ $\left(H_{0}^{1}(\Omega)\right)^{n} \int_{\Omega}\left[\varepsilon(u) D \varepsilon\left(v_{c}\right)+\operatorname{div} \sigma \cdot v_{c}\right] d \Omega=0$ implies that $\sum_{i} \oint_{\partial \Omega_{i}}(\sigma \cdot \vec{n}) \cdot v_{c} d s=0$, (2.6), (2.7) is equivalent to the following saddle point formulation:

$$
\begin{gathered}
\sum_{i}\left[\int_{\Omega_{i}} \tau \cdot D^{-1} \sigma d \Omega-\int_{\Omega_{i}} \tau \cdot \varepsilon(u) d \Omega+\oint_{\partial \Omega_{i}}(\tau \cdot \vec{n}) \cdot\left(u-u_{c}\right) d s\right]=0 \quad \forall \tau \in \mathbf{V}, \\
\sum_{i}\left[\int_{\Omega_{i}} \sigma \cdot \varepsilon(v) d \Omega-\oint_{\partial \Omega_{i}}(\sigma \cdot \vec{n}) \cdot\left(v-v_{c}\right) d s\right]=(f, v) \quad \forall\left(v, v_{c}\right) \in \mathbf{U} \times \mathbf{U}_{c}
\end{gathered}
$$

Then the first part of the theorem is proved, and the second formulation can be derived in the same way from the second order equation $-\operatorname{div} D \varepsilon(u)=f$.

Remark 2.1. In mechanics, the problem (2.2), (2.3) without the decomposition of $\Omega$ is referred to as Hellinger-Reissner principle. Pian's hybrid method [6, 9, 10] is based on (2.2), (2.3). Tong's hybrid method is based on (2.4), (2.5). In addition, we can point out that $(2.2),(2.3)$ and $(2.4),(2.5)$ are, respectively, the optimality conditions of the following saddle point problems:

$$
\inf _{\tau \in \mathbf{V}} \sup _{\left(v, v_{c}\right) \in \mathbf{U} \times \mathbf{U}_{c}}\left\{\frac{1}{2} \mathbf{a}(\tau, \tau)-\mathbf{b}_{2}(\tau, v)+\mathbf{b}_{1}\left(\tau, v-v_{c}\right)+(f, v)\right\}
$$

and

$$
\inf _{\left(v, v_{c}\right) \in \mathbf{U} \times \mathbf{U}_{c}} \sup _{\tau \in \mathbf{V}}\left\{\frac{1}{2} \mathbf{d}(v, v)-\mathbf{b}_{1}\left(\tau, v-v_{c}\right)-(f, v)\right\} .
$$

In the present paper, we do not directly discretize the above two saddle point problems that require the Babuska-Brezzi condition. Motivated by incorporating the second order terms $\mathbf{a}(\tau, \tau)$ and $d(v, v)$ into one system so that the inf-sup condition is weakened or circumvented, we will study the following problem.

Find $\left(\sigma, u, u_{c}\right) \in \mathbf{V} \times \mathbf{U} \times \mathbf{U}_{c}$ such that

$$
\begin{array}{ll}
\alpha \mathbf{a}(\sigma, \tau)-\alpha \mathbf{b}_{2}(\tau, u)+\mathbf{b}_{1}\left(\tau, u-u_{c}\right)=0 & \forall \tau \in \mathbf{V}, \\
\alpha \mathbf{b}_{2}(\sigma, v)-\mathbf{b}_{1}\left(\sigma, v-v_{c}\right)+(1-\alpha) \mathbf{d}(u, v)=(f, v) & \forall\left(v, v_{c}\right) \in \mathbf{U} \times \mathbf{U}_{c},
\end{array}
$$

which can be viewed as a weighted average of (2.2),(2.3) and (2.4),(2.5) with the weight factor $\alpha: 0<\alpha<1$.

In order to clearly display the enhanced stability of the combined variational principle (2.8),(2.9) and to learn how to circumvent the inf-sup condition, we will study the well-posedness of the problem (2.8),(2.9) in an abstract framework.

$\mathbf{V}$ and $\mathbf{U} \times \mathbf{U}_{c}$ can be equipped with the norms

$$
\begin{gathered}
\|\tau\|_{\mathbf{v}}=\left[\|\tau\|_{0, \Omega}^{2}+\sum_{\Omega_{i} \in \mathrm{T}_{h}} h_{i}^{2}\|\operatorname{div} \tau\|_{0, \Omega_{i}}^{2}\right]^{\frac{1}{2}}, \\
\left\|\left(v, v_{c}\right)\right\|_{\mathbf{U} \times \mathbf{U}_{c}}^{*}=\left[\sum_{\Omega_{i} \in \mathrm{T}_{h}}\left(\|\varepsilon(v)\|_{0, \Omega_{i}}^{2}+\left\|v-v_{c}\right\|_{\frac{1}{2}, \partial \Omega_{i}}^{2}\right)\right]^{\frac{1}{2}},
\end{gathered}
$$

where $\|v\|_{\frac{1}{2}, \partial \Omega_{i}}=\inf _{w \in\left(H_{0}^{1}\left(\Omega_{i}\right)\right)^{n}}\left[h_{i}^{-2}\|v+w\|_{0, \Omega_{i}}^{2}+\|\varepsilon(v+w)\|_{0, \Omega_{i}}^{2}\right]^{\frac{1}{2}}$, and $h_{i}$ denotes the diameter of $\Omega_{i}$.

For the validity of the second norm, it is merely needed to check that

$$
\left\|\left(v, v_{c}\right)\right\|_{\mathbf{U} \times \mathbf{U}_{c}}^{*}=0 \rightarrow v=v_{c}=0 .
$$


In fact, $\sum_{i}\left\|v-v_{c}\right\|_{\frac{1}{2}, \partial \Omega_{i}}^{2}=0$ implies $v \in\left(H_{0}^{1}(\Omega)\right)^{n}$. Hence, Korn's inequality can be used to conclude that $\|\varepsilon(v)\|=0 \rightarrow v=0$. Then, $\mathbf{V}$ and $\mathbf{U} \times \mathbf{U}_{c}$ are two Hilbert spaces.

Theorem 2.2. For the problem (2.8), (2.9), there exists a unique solution $\left(\sigma, u, u_{c}\right) \in$ $\mathbf{V} \times \mathbf{U} \times \mathbf{U}_{c}$ and $u \in\left(H_{0}^{1}(\Omega)\right)^{n},\left.\left(u-u_{c}\right)\right|_{\partial \Omega_{i}}=0 \forall \Omega_{i} \in \mathrm{T}_{h}$, and $\sigma=D[\varepsilon(u)]$, div $\sigma+$ $f=0$. In other words, for any $\alpha: 0<\alpha<1$, the system (2.8), (2.9) is another variational formulation of problem (2.1) that admits nonconforming displacements and piecewise discontinuous stresses.

In the proof of this theorem we need two lemmas. So, let us introduce the closed subspace of piecewise rigid displacements

$$
\mathbf{U}_{d}=\{v \in \mathbf{U} \mid: \mathbf{d}(v, v)=0\},
$$

and the linear mapping $G: \mathbf{U} \rightarrow \mathbf{U}_{d}$,

$$
\left.G(v)\right|_{\Omega_{i}}= \begin{cases}0 & \text { if } \Omega_{i} \in B\left(\mathrm{~T}_{h}\right), \\ R(v)+\frac{1}{2} R(\operatorname{curl} v) \times(X-R(X)) & \text { if } \Omega_{i} \notin B\left(\mathrm{~T}_{h}\right),\end{cases}
$$

where $B\left(\mathrm{~T}_{h}\right):=\left\{\left.\Omega_{i} \in \mathrm{T}_{h}\right|_{:}(n-1)-\operatorname{meas}\left(\partial \Omega_{i} \cap \partial \Omega\right)>0\right\}$ is a set of the boundary subdomains. $R$ and $X$ are two $n$-dimensional vectors. $X$ denotes the coordinate vector of any point $X \in \Omega, R(w)=\left(\operatorname{meas}\left(\Omega_{i}\right)\right)^{-1} \int_{\Omega_{i}} w d \Omega$.

Lemma 2.1. $G(v)=v \forall v \in \mathbf{U}_{d}$ and there exists a constant $C>0$, independent of $h_{i}$, such that for $v \in \mathbf{U}$

$$
\|v-G(v)\|_{t, \Omega_{i}} \leq C h_{i}^{1-t}\|\varepsilon(v)\|_{0, \Omega_{i}}(t=0,1)
$$

and

$$
\|v-G(v)\|_{\frac{1}{2}, \partial \Omega_{i}} \leq C\|\varepsilon(v)\|_{0, \Omega_{i}} .
$$

Proof. Since $v \in \mathbf{U}_{d}$ can be expressed by $\left.v\right|_{\Omega_{i}}=C_{1}+C_{2} \times X$, where $C_{1}, C_{2}$ are two $n$-dimensional constant vectors, it follows that $\frac{1}{2} R\left(\operatorname{curl}\left(C_{2} \times X\right)\right)=C_{2}$ and $R\left(C_{1}+C_{2} \times X\right)-C_{2} \times R(X)=C_{1}$. Then the first conclusion is proved.

Next, by the well-known error estimates for the interpolated approximation [2], it can easily be derived from the definition of $G(v)$ that for $w \in \mathbf{U}$,

$$
\begin{aligned}
& \|w-G(w)\|_{0, \Omega_{i}} \\
& =\|\left[w-\left(\operatorname{meas}\left(\Omega_{i}\right)\right)^{-1} \int_{\Omega_{i}} w d \Omega\right]+\frac{1}{2} R(\operatorname{curl} w) \\
& \times\left[\left(\operatorname{meas}\left(\Omega_{i}\right)\right)^{-1} \int_{\Omega_{i}} X d \Omega-X\right] \|_{0, \Omega_{i}} \\
& \leq C h_{i}|w|_{1, \Omega_{i}}+\frac{1}{2}|R(\operatorname{curl} w)| C h_{i}\left[\operatorname{meas}\left(\Omega_{i}\right)\right]^{\frac{1}{2}} \leq C h_{i}|w|_{1, \Omega_{i}},
\end{aligned}
$$

where the constant $C>0$ is independent of $h_{i}$.

Since $G(v-G(v))=G(v)-G^{2}(v)=0$, it follows by setting up $w=v-G(v)$ in the above estimation that

$$
\|v-G(v)\|_{0, \Omega_{i}}=\|v-G(v)-G(v-G(v))\|_{0, \Omega_{i}} \leq C h_{i}|v-G(v)|_{1, \Omega_{i}} .
$$

On the other hand, by virtue of the definition of $G(v)$, we get

$$
\int_{\Omega_{i}} \operatorname{curl}(G(v)-v) d \Omega=0
$$


which enables us to apply Korn's inequality to $G(v)-v$ (see [7] §42, Thm. 2]). Thus we have

$$
|v-G(v)|_{1, \Omega_{i}} \leq C\|\varepsilon(v-G(v))\|_{0, \Omega_{i}} \leq C\|\varepsilon(v)\|_{0, \Omega_{i}},
$$

where $C>0$ is independent of $h_{i}$. Combining this estimation with (2.10) leads to the required second results. The proof is completed.

Lemma 2.2. Let us define

$$
\left\|\left(v, v_{c}\right)\right\|_{\mathbf{U} \times \mathbf{U}_{c}}=\left[\sum_{\Omega_{i} \in \mathbf{T}_{h}}\left(\|\varepsilon(v)\|_{0, \Omega_{i}}^{2}+\left\|G(v)-v_{c}\right\|_{\frac{1}{2}, \partial \Omega_{i}}^{2}\right)\right]^{\frac{1}{2}} .
$$

Then $\|(\cdot, \cdot)\|_{\mathbf{U} \times \mathbf{U}_{c}}$ is a norm equivalent to $\|(\cdot, \cdot)\|_{\mathbf{U}_{\times} \mathbf{U}_{c}}$; that is, there exists a constant $C>0$, independent of $h_{i}$, such that for $\left(v, v_{c}\right) \in \mathbf{U} \times \mathbf{U}_{c}$,

$$
C^{-1}\left\|\left(v, v_{c}\right)\right\|_{\mathbf{U} \times \mathbf{U}_{c}} \leq\left\|\left(v, v_{c}\right)\right\|_{\mathbf{U} \times \mathbf{U}_{c}}^{*} \leq C\left\|\left(v, v_{c}\right)\right\|_{\mathbf{U} \times \mathbf{U}_{c}} .
$$

Proof. By virtue of Lemma 2.1 there exists a constant $C$ such that for $\left(v, v_{c}\right) \in$ $\mathbf{U} \times \mathbf{U}_{c}$,

$$
\begin{aligned}
\left\|v-v_{c}\right\|_{\frac{1}{2}, \partial \Omega_{i}} & \leq\left\|G(v)-v_{c}\right\|_{\frac{1}{2}, \partial \Omega_{i}}+\|v-G(v)\|_{\frac{1}{2}, \partial \Omega_{i}} \\
& \leq\left\|G(v)-v_{c}\right\|_{\frac{1}{2}, \partial \Omega_{i}}+C\|\varepsilon(v)\|_{0, \Omega_{i}} .
\end{aligned}
$$

By the definitions of $\|(\cdot, \cdot)\|_{\mathbf{U}_{\times} \mathbf{U}_{c}}^{*}$ and $\|(\cdot, \cdot)\|_{\mathbf{U} \times \mathbf{U}_{c}}$, this inequality yields the desired result, i.e., $\left\|\left(v, v_{c}\right)\right\|_{\mathbf{U} \times \mathbf{U}_{c}}^{*} \leq C\left\|\left(v, v_{c}\right)\right\|_{\mathbf{U} \times \mathbf{U}_{c}}$.

In the same way, we can conclude that the left-hand side inequality holds as well. Then, the lemma is proved.

Proof of Theorem 2.2 This proof is nothing less than a concrete application of Theorem 3.1 in [18]. For the sake of completeness, we sketch the proof here to give a clue to a new stabilized discretization of the problem (2.8),(2.9)).

First, it is easily derived from Lemma 2.1 that the orthogonal complementary space of $\mathbf{U}_{d} \times \mathbf{U}_{C}$, with respect to the norm $\|(\cdot, \cdot)\|_{\mathbf{U} \times \mathbf{U}_{C}}$, can be expressed as $\left(\mathbf{U}_{d} \times \mathbf{U}_{C}\right)^{\perp}=\mathbf{U}_{d}^{\perp} \times\{0\}$, where $\mathbf{U}_{d}^{\perp}:=\{v-G(v), \forall v \in \mathbf{U}\}=\{v \in \mathbf{U} \mid: G(v)=0\}$. Thus, we can express $\left(v, v_{c}\right) \in \mathbf{U}_{d} \times \mathbf{U}_{C}$ by the orthogonal decomposition

$$
\left(v, v_{c}\right)=\left(v_{d}, v_{c}\right) \oplus\left(v_{0}, 0\right),
$$

where $v_{0}=v-v_{d}, v_{d}=G(v)$. Based on this decomposition, the problem (2.8), (2.9) can be written as follows:

Find $\left(\left(\sigma, u_{0}\right),\left(u_{d}, u_{c}\right)\right) \in\left(\mathbf{V} \times \mathbf{U}_{d}^{\perp}\right) \times\left(\mathbf{U}_{d} \times \mathbf{U}_{c}\right)$ such that

$$
\begin{aligned}
\alpha \mathbf{a}(\sigma, \tau)-\mathbf{b}_{\alpha}\left(\tau, u_{0}\right)+\mathbf{b}_{1}\left(\tau, u_{d}-u_{c}\right) & =0 & & \forall \tau \in \mathbf{V}, \\
\mathbf{b}_{\alpha}\left(\sigma, v_{0}\right)+(1-\alpha) \mathbf{d}\left(u_{0}, v_{0}\right) & =\left(f, v_{0}\right) & & \forall v_{0} \in \mathbf{U}_{d}^{\perp}, \\
-\mathbf{b}_{1}\left(\sigma, v_{d}-v_{c}\right) & =\left(f, v_{d}\right) & & \forall\left(v_{d}, v_{c}\right) \in \mathbf{U}_{d} \times \mathbf{U}_{c},
\end{aligned}
$$

where $\mathbf{b}_{\alpha}\left(\tau, v_{0}\right)=\alpha \mathbf{b}_{2}\left(\tau, v_{0}\right)-\mathbf{b}_{1}\left(\tau, v_{0}\right)$.

For the equation (2.13), it follows from Lemmas 2.1 and 2.2 that for $\left(\tau, v_{0}\right) \in$ $\mathbf{V} \times \mathbf{U}_{d}^{\perp}\left(v_{0}=v-G(v)\right)$,

$$
\left|\mathbf{b}_{\alpha}\left(\tau, v_{0}\right)\right| \leq\|\tau\|_{\mathbf{V}} \cdot\left\|\left(v_{0}, 0\right)\right\|_{\mathbf{U} \times \mathbf{U}_{c}}^{*} \leq C\|\tau\|_{\mathbf{V}} \cdot\left\|\left(v_{0}, 0\right)\right\|_{\mathbf{U} \times \mathbf{U}_{c}}
$$


and

$$
\begin{aligned}
\left\|\left(v_{0}, 0\right)\right\|_{\mathbf{U} \times \mathbf{U}_{c}} & =\left[\sum_{i}\left(\left\|\varepsilon\left(v_{0}\right)\right\|_{0, \Omega_{i}}^{2}+\left\|G\left(v_{0}\right)\right\|_{\frac{1}{2}, \partial \Omega_{i}}^{2}\right)\right]^{\frac{1}{2}}=\left[\sum_{i}\left\|\varepsilon\left(v_{0}\right)\right\|_{0, \Omega_{i}}^{2}\right]^{\frac{1}{2}} \\
& \leq C \mathbf{d}^{\frac{1}{2}}\left(v_{0}, v_{0}\right),
\end{aligned}
$$

which say that $\mathbf{b}_{\alpha}\left(\tau, v_{0}\right)$ is bounded on $\mathbf{V} \times\left(\mathbf{U}_{d}^{\perp} \times\{0\}\right)$, and $\mathbf{d}(\cdot, \cdot)$ is $\mathbf{U}_{d}^{\perp} \times\{0\}$ elliptic. Then, by virtue of the Lax-Milgram theorem, there exists a resolvent operator $T: \mathbf{V} \rightarrow \mathbf{U}_{d}^{\perp}$ such that

$$
(1-\alpha) \mathbf{d}\left(T(\tau), v_{0}\right)=-\mathbf{b}_{\alpha}\left(\tau, v_{0}\right) \quad \forall v_{0} \in \mathbf{U}_{d}^{\perp}
$$

and

$$
\|(T(\tau), 0)\|_{\mathbf{U} \times \mathbf{U}_{c}} \leq \frac{C^{*}}{1-\alpha}\left\|\left(\alpha B_{2}-B_{1}\right) \tau\right\|_{\left(\mathbf{U} \times \mathbf{U}_{c}\right)^{\prime}},
$$

where $\left(\mathbf{U} \times \mathbf{U}_{C}\right)^{\prime}$ denotes the dual spaces of $\mathbf{U} \times \mathbf{U}_{c}$, and $B_{2}$ and $B_{1}$ denote two linear operators defined respectively by

$$
\left.\begin{array}{rl}
\mathbf{b}_{2}(\tau, v) & =\left\langle B_{2}(\tau),(v, 0)\right\rangle_{\left(\mathbf{U} \times \mathbf{U}_{c}\right)^{\prime} \times\left(\mathbf{U} \times \mathbf{U}_{c}\right)} \\
\mathbf{b}_{1}\left(\tau, v-v_{c}\right) & =\left\langle B_{1}(\tau),\left(v, v_{c}\right)\right\rangle_{\left(\mathbf{U} \times \mathbf{U}_{c}\right)^{\prime} \times\left(\mathbf{U} \times \mathbf{U}_{c}\right)}
\end{array}\right\} \quad \forall\left(\tau,\left(v, v_{c}\right)\right) \in \mathbf{V} \times\left(\mathbf{U} \times \mathbf{U}_{c}\right) .
$$

In addition, there exists a unique solution $u_{f}^{\perp} \in \mathbf{U}_{d}^{\perp}$ such that

$$
(1-\alpha) \mathbf{d}\left(u_{f}^{\perp}, v_{0}\right)=\left(f, v_{0}\right) \quad \forall v_{0} \in \mathbf{U}_{d}^{\perp} .
$$

Thus the solution $u_{0}$ for 2.13) can be expressed by

$$
u_{0}=T(\sigma)+u_{f}^{\perp}
$$

and we have

$$
\begin{aligned}
-\mathbf{b}_{\alpha}\left(\tau, u_{0}\right) & =(1-\alpha) \mathbf{d}\left(T(\tau), T(\sigma)+u_{f}^{\perp}\right) \\
& =(1-\alpha) \mathbf{d}(T(\sigma), T(\tau))+(f, T(\tau)),
\end{aligned}
$$

which enables the problem (2.12),(2.13),(2.14) to reduce into the following.

Find $\left(\sigma, u_{d}, u_{c}\right) \in \mathbf{V} \times \mathbf{U}_{d} \times \mathbf{U}_{c}$ such that

$$
\begin{aligned}
\alpha \mathbf{a}(\sigma, \tau) & +(1-\alpha) \mathbf{d}(T(\sigma), T(\tau)) & & \\
& +\mathbf{b}_{1}\left(\tau, u_{d}-u_{c}\right)=-(f, T(\tau)) & & \forall \tau \in \mathbf{V}, \\
& -\mathbf{b}_{1}\left(\sigma, v_{d}-v_{c}\right)=\left(f, v_{d}\right) & & \forall\left(v_{d}, v_{c}\right) \in \mathbf{U}_{d} \times \mathbf{U}_{c} .
\end{aligned}
$$

This is a stability-enhanced saddle point problem. By virtue of Theorem II-1.1 in [1], the existence and uniqueness of the solution $\left(\sigma, u_{d}, u_{c}\right)$ can be guaranteed by checking the corresponding inf-sup condition and the kernel-ellipticity condition.

To the end, we equip the space $\mathbf{V}$ with another norm defined by

$$
\|\tau\|_{\mathbf{V}}^{*}:=\left[\alpha \mathbf{a}(\tau, \tau)+\frac{1}{1-\alpha}\left\|\left(\alpha B_{2}-B_{1}\right) \tau\right\|_{\left(\mathbf{U} \times \mathbf{U}_{c}\right)^{\prime}}^{2}\right]^{\frac{1}{2}} .
$$


By Lemma 2.2 and Schwarz inequality, we have

$$
\begin{aligned}
\left\|\left(\alpha B_{2}-B_{1}\right) \tau\right\|_{\left(\mathbf{U} \times \mathbf{U}_{c}\right)^{\prime}} & =\sup _{\left(v, v_{c}\right) \neq 0} \frac{\alpha \mathbf{b}_{2}(\tau, v)-\mathbf{b}_{1}\left(\tau, v-v_{c}\right)}{\left\|\left(v, v_{c}\right)\right\|_{\mathbf{U} \times \mathbf{U}_{c}}} \\
& \leq C \sup _{\left(v, v_{c}\right) \neq 0} \frac{\alpha \mathbf{b}_{2}(\tau, v)-\mathbf{b}_{1}\left(\tau, v-v_{c}\right)}{\left\|\left(v, v_{c}\right)\right\|_{\mathbf{U} \times \mathbf{U}_{c}}^{*}} \\
& \leq C\|\tau\|_{\mathbf{V}} .
\end{aligned}
$$

Then

$$
\|\tau\|_{\mathbf{v}}^{*} \leq C\|\tau\|_{\mathbf{v}}
$$

By the definition of $\|\cdot\|_{\mathbf{V}}^{*}$, it is obvious that $\mathbf{b}_{1}\left(\tau, v-v_{c}\right)$ is bounded on $\mathbf{V} \times \mathbf{U}_{d} \times \mathbf{U}_{c}$, i.e.,

$$
\left|\mathbf{b}_{1}\left(\tau, v-v_{c}\right)\right| \leq C\|\tau\|_{\mathbf{V}}^{*} \cdot\left\|\left(v, v_{c}\right)\right\|_{\mathbf{U} \times \mathbf{U}_{c}} \quad \forall\left(\tau, v, v_{c}\right) \in \mathbf{V} \times \mathbf{U}_{d} \times \mathbf{U}_{c} .
$$

Thus, recalling that $\tau \cdot \vec{n}: H\left(\operatorname{div} ; \Omega_{i}\right) \rightarrow H^{-\frac{1}{2}}\left(\partial \Omega_{i}\right)$ is surjective, we have

$$
\begin{aligned}
\sup _{\tau \neq 0} \frac{\mathbf{b}_{1}\left(\tau, v_{d}-v_{c}\right)}{\|\tau\|_{\mathbf{V}}^{*}} & \geq C \sup _{\tau \neq 0} \frac{\mathbf{b}_{1}\left(\tau, v_{d}-v_{c}\right)}{\|\tau\|_{\mathbf{V}}} \\
& \geq C\left[\sum_{i}\left\|\left(v_{d}-v_{c}\right)\right\|_{\frac{1}{2}, \partial \Omega_{i}}^{2}\right]^{\frac{1}{2}} \\
& \geq C\left\|\left(v_{d}, v_{c}\right)\right\|_{\mathbf{U} \times \mathbf{U}_{c}}^{*} \\
& \geq C\left\|\left(v_{d}, v_{c}\right)\right\|_{\mathbf{U} \times \mathbf{U}_{c}} \quad \forall\left(v_{d}, v_{c}\right) \in \mathbf{U}_{d} \times \mathbf{U}_{c},
\end{aligned}
$$

that is, the inf-sup condition is verified.

Now we return to prove that there exists a constant $C>0$, independent of $h_{i}$, such that

$$
\alpha \mathbf{a}(\tau, \tau)+(1-\alpha) \mathbf{d}(T(\tau), T(\tau)) \geq C\|\tau\|_{\mathbf{V}}^{*} \quad \forall \tau \in \operatorname{ker}\left(B_{1}\right),
$$

where $\operatorname{ker}\left(B_{1}\right):=\left\{\tau \in \mathbf{V} \mid: \mathbf{b}_{1}\left(\tau, v_{d}-v_{c}\right)=0, \forall\left(v_{d}, v_{c}\right) \in \mathbf{U}_{d} \times \mathbf{U}_{c}\right\}$.

In fact, since $\mathbf{b}_{2}\left(\tau, v_{d}\right)=0, \forall \tau \in \mathbf{V}$, it follows by $\mathbf{U} \times \mathbf{U}_{c}=\left(\mathbf{U}_{d} \times \mathbf{U}_{c}\right) \oplus\left(\mathbf{U}_{d}^{\perp} \times\{0\}\right)$ that for $\tau \in \operatorname{ker}\left(B_{1}\right)$,

$$
\left\langle\left(\alpha B_{2}-B_{1}\right) \tau,\left(v_{d}, v_{c}\right)\right\rangle_{\left(\mathbf{U} \times \mathbf{U}_{c}\right)^{\prime} \times\left(\mathbf{U} \times \mathbf{U}_{c}\right)}=0 \quad \forall\left(v_{d}, v_{c}\right) \in \mathbf{U}_{d} \times \mathbf{U}_{c},
$$

then

$$
\begin{aligned}
\left\|\left(\alpha B_{2}-B_{1}\right) \tau\right\|_{\left(\mathbf{U} \times \mathbf{U}_{c}\right)^{\prime}} & =\sup _{\substack{v_{0} \in \mathbf{U}_{d}^{\perp} \\
v_{0} \neq 0}} \frac{\left\langle\left(\alpha B_{2}-B_{1}\right) \tau,\left(v_{0}, 0\right)\right\rangle_{\left(\mathbf{U} \times \mathbf{U}_{c}\right)^{\prime} \times\left(\mathbf{U} \times \mathbf{U}_{c}\right)}}{\left\|\left(v_{0}, 0\right)\right\|_{\mathbf{U} \times \mathbf{U}_{c}}} \\
& =\sup _{v_{0} \neq 0} \frac{(1-\alpha) \mathbf{d}\left(T(\tau), v_{0}\right)}{\left(\sum_{i}\left\|\varepsilon\left(v_{0}\right)\right\|_{0, \Omega_{i}}^{2}\right)^{\frac{1}{2}}} \\
& \leq(1-\alpha) \mathbf{d}^{\frac{1}{2}}(T(\tau), T(\tau)) \sup _{v_{0} \neq 0} \frac{\mathbf{d}^{\frac{1}{2}}\left(v_{0}, v_{0}\right)}{\left(\sum_{i}\left\|\varepsilon\left(v_{0}\right)\right\|_{0, \Omega_{i}}^{2}\right)^{\frac{1}{2}}} \\
& \leq C(1-\alpha) \mathbf{d}^{\frac{1}{2}}(T(\tau), T(\tau)) .
\end{aligned}
$$


Hence,

$$
\begin{aligned}
\left(\|\tau\|_{\mathbf{V}}^{*}\right)^{2} & =\alpha \mathbf{a}(\tau, \tau)+\frac{1}{1-\alpha}\left\|\left(\alpha B_{2}-B_{1}\right) \tau\right\|_{\left(\mathbf{U} \times \mathbf{U}_{c}\right)^{\prime}}^{2} \\
& \leq C^{2}[\alpha \mathbf{a}(\tau, \tau)+(1-\alpha) \mathbf{d}(T(\tau), T(\tau))] ;
\end{aligned}
$$

that is, the bilinear form $\alpha \mathbf{a}(\tau, \sigma)+(1-\alpha) \mathbf{d}(T(\sigma), T(\tau))$ in (2.16) is $\operatorname{ker}\left(B_{1}\right)$-coercive.

Up to now, all the conditions are satisfied which are required by the BabuskaBrezzi theory [11], thus the existence and uniqueness of the solution $\left(\sigma, u_{d}^{\perp}+u_{d}, u_{c}\right)$, is proved. Moreover, the other results in Theorem 2.2 can be obtained by the standard variational calculus arguments. Then the theorem is proved.

In a word, Theorem 2.2 provides us with a new discretization approach to hybrid schemes. This analysis shows that in the case of $\mathbf{U}_{C}$ being completely independent of $\mathbf{U}$, only the inf-sup condition (2.19) is required; i.e., for $\left(v, v_{c}\right) \in\left(U \times U_{C}\right)_{d}:=$ $\left\{\left(v, v_{c}\right) \in \mathbf{U} \times \mathbf{U}_{C}: d(v, v)=0\right\}$,

$$
\sup _{\tau \in \mathbf{V}} \frac{b_{1}\left(\tau, v-v_{c}\right)}{\|\tau\|_{\mathbf{V}}} \geq C\left\|\left(v, v_{c}\right)\right\|_{\mathbf{U} \times \mathbf{U}_{C}} .
$$

In the next section we will point out that if associating $\mathbf{U}_{c}$ with $\mathbf{U}$ so that $\mathbf{U}$ is a strain-enriched space of $\mathbf{U}_{c}$, this condition can be circumvented. A combined hybrid method of avoiding this inf-sup condition can be presented.

\section{HybRid Finite ELEMENT SCHEMES OF AVOIDING THE INF-SUP CONDITION}

From the proof of Theorem 2.1, it is known that $v_{c} \in\left(H_{0}^{1}(\Omega)\right)^{n}$ may not be independent of $v \in \mathbf{U}$. We can consider another type of the combined variational principle that $\mathbf{U}_{C}$ is associated with $\mathbf{U}$. In fact, let $\overline{\mathbf{U}}$ be a subspace of $\mathbf{U}$, $\overline{\mathbf{U}} \supset\left(H_{0}^{1}(\Omega)\right)^{n}$, and let $T_{c}(v): \overline{\mathbf{U}} \rightarrow\left(H_{0}^{1}(\Omega)\right)^{n}$ be a linear mapping such that

$$
T_{c}(v)=v \quad \forall v \in\left(H_{0}^{1}(\Omega)\right)^{n} ;
$$

there exists a constant $C>0$, such that for $(\tau, v) \in \mathbf{V} \times \overline{\mathbf{U}}$,

$$
\left|b_{1}\left(\tau, v-T_{c}(v)\right)\right| \leq C\|\tau\|_{\mathbf{v}} \cdot d^{\frac{1}{2}}(v, v) .
$$

By replacing the displacement space $\mathbf{U} \times \mathbf{U}_{C}$ with $\mathbf{U} \times T_{c}(\overline{\mathbf{U}})$, the variational formulation (2.8), (2.9) is changed into the following.

Find $(\sigma, u) \in \mathbf{V} \times \overline{\mathbf{U}}$ such that

$$
\begin{aligned}
\alpha a(\sigma, \tau)-b_{\alpha}(\tau, u) & =0 & & \forall \tau \in \mathbf{V}, \\
b_{\alpha}(\sigma, v)+(1-\alpha) d(u, v) & =(f, v) & & \forall v \in \overline{\mathbf{U}},
\end{aligned}
$$

where $b_{\alpha}(\tau, v):=\alpha b_{2}(\tau, v)-b_{1}\left(\tau, v-T_{c}(v)\right)$.

Two displacement variables are reduced into one. For convenience, $T_{c}(\cdot)$ will be referred to as a coupling operator.

In this case, the additional assumption that

$$
\{v \in \overline{\mathbf{U}}: d(v, v)=0\}=: \overline{\mathbf{U}}_{d}=\{0\}
$$

enables us to equip the space $\overline{\mathbf{U}}$ with the norm $\|v\|_{\mathbf{U}}:=d^{\frac{1}{2}}(v, v)$. Thus, since $b_{\alpha}(\tau, v)$ is a bounded bilinear form on $\mathbf{V} \times \overline{\mathbf{U}}$ and $\left|b_{\alpha}(\tau, v)\right| \leq C\|\tau\|_{\mathbf{V}}\|v\|_{\mathbf{U}}$, by virtue of the resolvent operator $T(\tau): \mathbf{V} \rightarrow \overline{\mathbf{U}}$ previously defined by

$$
(1-\alpha) d(T(\tau), v)=-b_{\alpha}(\tau, v) \quad \forall v \in \overline{\mathbf{U}}
$$


the same treatment as in the proof of Theorem 2.2 makes the problem (3.3), (3.4) reduce into the following.

Find $\sigma \in \mathbf{V}$ such that

$$
\alpha a(\sigma, \tau)+(1-\alpha) d(T(\sigma), T(\tau))=-(f, T(\tau)) \quad \forall \tau \in \mathbf{V},
$$

which is merely an extreme value problem.

In contrast with the saddle point problem (2.16), (2.17) the inf-sup condition (2.20) is avoided in this case, but by the same argument as before, we can still conclude that the problem (3.5) is $\mathbf{V}$-coercive. Then the following lemma is proved:

Lemma 3.1. Assume that

$$
\begin{aligned}
& T_{c}(v) \text { is a coupling operator; } \\
& \text { for } v \in \overline{\mathbf{U}}, d(v, v)=0 \rightarrow v=0 .
\end{aligned}
$$

Then thee exists a unique solution $(\sigma, u) \in \mathbf{V} \times \overline{\mathbf{U}}$ of the problem (3.3), (3.4) such that $\operatorname{div}(\sigma)+f=0$ and $\sigma=D \varepsilon(v)$, and the inf-sup condition (2.20) is avoided.

Now we focus our attention on a class of particular piecewise $H^{1}$-spaces, including the so-called strain-enriched displacement spaces used in 11-14. Let $\mathbf{U}_{I}$ be a finite dimensional space of nonconforming bubble type, such that

$$
\begin{aligned}
& \mathbf{U}_{I} \cap\left(H_{0}^{1}(\Omega)\right)^{n}=\{0\}, \\
& \left\|v_{I}\right\|_{0, \Omega_{i}} \leq C h_{i}\left\|\varepsilon\left(v_{I}\right)\right\|_{0, \Omega_{i}} \quad \forall v_{I} \in \mathbf{U}_{I},
\end{aligned}
$$

where $C$ is independent of $h_{i}$.

For practical purposes, the following spaces can be viewed as typical examples of this type:

$$
\left.\mathbf{U}_{I}^{(1)}\right|_{\Omega_{i}}:=\left\{\hat{v}_{I} \circ F_{i}^{-1}: \hat{v}_{I} \in \operatorname{span}\left(1-\xi^{2}, 1-\eta^{2}\right)\right\},
$$

where $F_{i}(\xi, \eta)$ denotes the affine mapping from the referential square $\{(\xi, \eta) \in$ $[-1,1] \times[-1,1]\}$ to the quadrilateral $\Omega_{i}$;

$\left.\mathbf{U}_{I}^{(2)}\right|_{\Omega_{i}}:=\left\{\hat{v}_{I} \circ F_{i}^{-1}: \hat{v}_{I} \in \operatorname{span}\left(1-\xi^{2}, 1-\eta^{2}, \xi\left(1-\xi^{2}\right), \eta\left(1-\xi^{2}\right), \xi\left(1-\eta^{2}\right), \eta\left(1-\eta^{2}\right)\right)\right\}$

and so on, as well as the corresponding three-dimensional counterparts. It can be shown by Proposition 3.1 below that the conditions (3.8), (3.9) are satisfied for all these spaces. Following the mechanics, $\overline{\mathbf{U}}:=\left(H_{0}^{1}(\Omega)\right)^{n} \oplus \mathbf{U}_{I}$ will be referred to as a strain-enriched space.

Due to the assumptions (3.8), (3.9) $), v_{I} \in \mathbf{U}_{I}$ and $v_{I} \neq 0$ imply that $d\left(v_{I}, v_{I}\right) \neq 0$ and $d\left(v_{I}-v_{I}^{*}, v_{I}-v_{I}^{*}\right) \neq 0$, where $v_{I}^{*} \in\left(H_{0}^{1}(\Omega)\right)^{n}$ denotes the orthogonal projector of $v_{I}$ on $\left(H_{0}^{1}(\Omega)\right)^{n}$ with respect to the norm $d^{\frac{1}{2}}(v, v)$; i.e.,

$$
d\left(v_{I}-v_{I}^{*}, w\right)=0 \quad \forall w \in\left(H_{0}^{1}(\Omega)\right)^{n} .
$$

Thus, for $v \in \overline{\mathbf{U}}$ such that $v=v_{c}+v_{I}, v_{c} \in\left(H_{0}^{1}(\Omega)\right)^{n}, v_{I} \in \mathbf{U}_{I}$ and $v_{I} \neq 0$, we have an orthogonal decomposition of

$$
v=\left(v_{c}+v_{I}^{*}\right) \oplus\left(v_{I}-v_{I}^{*}\right)
$$

such that

$$
d(v, v) \geq d\left(v_{I}-v_{I}^{*}, v_{I}-v_{I}^{*}\right)>0 .
$$

Hence, $d(v, v)=0$ yields that $v_{I}=0$ and $v=v_{c} \in\left(H_{0}^{1}(\Omega)\right)^{n}$, then $v_{c}=0$ due to Korn's inequality. Based on this, the first conclusion of the following theorem is proved. 
Theorem 3.1. The strain-enriched space $\overline{\mathbf{U}}$ is a Hilbert space with norm $\|v\|_{\mathbf{U}}:=$ $d^{\frac{1}{2}}(v, v)$, and for $v=v_{c}+v_{I} \in \overline{\mathbf{U}}, \bar{T}_{C}(v)=v_{c}$ is a coupling operator. Therefore the variational formulation (3.3), (3.4) in which $\mathbf{U}$ and $T_{c}(\cdot)$ are replaced by $\overline{\mathbf{U}}$ and $\bar{T}_{c}(\cdot)$, respectively, is equivalent to (2.1), and the inf-sup condition corresponding to (2.20) is avoided.

Proof. It is obvious that $\bar{T}_{c}(\cdot)$ is linear and $\bar{T}_{c}(v)=v$ for $v \in\left(H_{0}^{1}(\Omega)\right)^{n}$. Therefore, for the second conclusion it remains to prove that there exists $C>0$ such that

$$
\left|b_{1}\left(\tau, v-\bar{T}_{c}(v)\right)\right| \leq C\|\tau\|_{\mathbf{v}} d^{\frac{1}{2}}(v, v) \quad \forall(\tau, v) \in \mathbf{V} \times \overline{\mathbf{U}} .
$$

In fact, by the assumption (3.9), we have

$$
\left\|v-\bar{T}_{c}(v)\right\|_{0, \Omega_{i}} \leq C h_{i}\left\|v-\bar{T}_{c}(v)\right\|_{\mathbf{U}} .
$$

Since $\mathbf{U}_{I}$ is finite dimensional and $d\left(v_{I}, v_{I}\right)>0$ implies that $d\left(v_{I}-v_{I}^{*}, v_{I}-v_{I}^{*}\right)>0$, we obtain

$$
\min _{\substack{v_{I} \in \mathbf{U}_{I} \\ v_{I} \neq 0}} \frac{d\left(v_{I}-v_{I}^{*}, v_{I}-v_{I}^{*}\right)}{d\left(v_{I}, v_{I}\right)}=C_{0}>0,
$$

where $C_{0}$ denotes the minimum characteristic value of the matrix

$$
\left[\frac{d\left(\bar{v}_{I}^{(t)}, \bar{v}_{I}^{(j)}\right)}{d^{\frac{1}{2}}\left(\bar{v}_{I}^{(t)}, \bar{v}_{I}^{(t)}\right) d^{\frac{1}{2}}\left(\bar{v}_{I}^{(j)}, \bar{v}_{I}^{(j)}\right)}\right]_{t, j},
$$

and $\bar{v}_{I}^{t}:=v_{I}^{(t)}-v_{I}^{*(t)},\left\{v_{I}^{(t)}\right\}_{t}$ is a complete set of linearly independent base functions. Thus by (3.10) and (3.12), we get

$$
C_{0}\left\|v-\bar{T}_{c}(v)\right\|_{\mathbf{U}}^{2} \leq\left\|v_{I}-v_{I}^{*}\right\|_{\mathbf{U}}^{2} \leq\|v\|_{\mathbf{U}}^{2} .
$$

By Green's formula and Schwarz's inequality,

$$
\begin{aligned}
\left|b_{1}\left(\tau, v-\bar{T}_{c}(v)\right)\right| & =\left|\sum_{i} \int_{\Omega_{i}}\left[\operatorname{div} \tau \cdot v_{I}+\tau \cdot \varepsilon\left(v_{I}\right)\right] d \Omega\right| \\
& \leq\|\tau\|_{\mathbf{v}} \cdot\left\{\sum_{i}\left[\frac{\left\|v_{I}\right\|_{0, \Omega_{i}}^{2}}{h_{i}^{2}}+\left\|\varepsilon\left(v_{I}\right)\right\|_{0, \Omega_{i}}^{2}\right]\right\}^{\frac{1}{2}} \\
& \leq C\left\|_{\tau}\right\|_{\mathbf{V}}\|v\|_{\mathbf{U}} \quad \text { due to (3.11) and (3.13). }
\end{aligned}
$$

Then $\bar{T}_{c}(\cdot)$ is a coupling operator. Therefore, by Lemma 3.1, the theorem is proved.

Now we turn to discuss the finite element approximations and convergence analysis. Let $\mathbf{V}^{h}$ and $\mathbf{U}^{h}$ be two finite element spaces such that $\mathbf{V}^{h} \subset \mathbf{V}$ and $\mathbf{U}^{h} \subset \mathbf{U}$

$$
\begin{aligned}
& \mathbf{V}^{h}:=\left\{\tau \in \mathbf{V}:\left.\tau\right|_{\Omega_{i}}=\hat{p}_{r} \circ F_{i}^{-1}, \forall \Omega_{i} \in T_{h}\right\}=: \mathbf{V}_{r}^{h}, \\
& \mathbf{U}^{h}:=\left\{v \in \mathbf{U}:\left.v\right|_{\Omega_{i}}=\hat{p}_{s} \circ F_{i}^{-1}, \forall \Omega_{i} \in T_{h}\right\},
\end{aligned}
$$

where $r$ and $s$ denote nonnegative integers, $\widehat{P}_{r}$ the set of polynomials of order $\leq r$, $F_{i}(\cdot)$ the affine mapping from a referential element $K$ to $\Omega_{i}$. Thus, the combined hybrid schemes can be established as follows. 
Find $\left(\sigma_{h}, u_{h}\right) \in \mathbf{V}^{h} \times \mathbf{U}^{h}$ such that

$$
\begin{aligned}
\alpha a\left(\sigma_{h}, \tau\right)-b_{\alpha}\left(\tau, u_{h}\right) & =0 & & \forall \tau \in \mathbf{V}^{h}, \\
b_{\alpha}\left(\sigma_{h}, v\right)+(1-\alpha) d\left(u_{h}, v\right) & =(f, v) & & \forall v \in \mathbf{U}^{h} .
\end{aligned}
$$

The existence and uniqueness of the hybrid finite element solution $\left(\sigma_{h}, u_{h}\right)$ is guaranteed by the argument in Theorem 3.1. Then from the following theorem, we can know that the hybrid scheme (3.14), (3.15) is stabilized.

Theorem 3.2. Assume that $\mathbf{U}^{h}$ is a strain-enriched subspace and there exists $C>0$, independent of $h_{i}$, such that for $\Omega_{i} \in \mathrm{T}_{h}$ and $v \in \mathbf{U}^{h}$,

$$
\left\|v-T_{c}(v)\right\|_{\frac{1}{2}, \partial \Omega_{i}} \leq C\|\varepsilon(v)\|_{0, \Omega_{i}} .
$$

Then

$$
\begin{aligned}
& \| \sigma- \sigma_{h}\left\|_{0, \Omega}+\left(\frac{1-\alpha}{\alpha}\right)^{\frac{1}{2}}(1-2 \nu)^{-1}\right\| u-u_{h} \|_{\mathbf{U}} \leq C[\alpha(1-\alpha)]^{-\frac{1}{2}} \\
& \cdot\left\{\inf _{\tau \in \mathbf{V}^{h}}\|\sigma-\tau\|_{\mathbf{V}}+(1-2 \nu)^{-1} \inf _{v \in \mathbf{U}^{h}}\left[\|u-v\|_{\mathbf{U}}+\sup _{\tau \in \mathbf{V}^{h}} \frac{\mathbf{b}_{1}\left(\tau, v-T_{c}(v)\right)}{\|\tau\|_{\mathbf{V}}}\right]\right\},
\end{aligned}
$$

where $\|v\|_{\mathbf{U}}:=\left(\sum_{\Omega_{i} \in \mathrm{T}_{h}}\|\varepsilon(v)\|_{0, \Omega_{i}}^{2}\right)^{\frac{1}{2}}$ and $C$ is independent of $h, \alpha$ and the Poisson ratio $\nu$.

Proof. Let us first assume that $\left(\Pi_{1} \sigma, \Pi_{0} u\right) \in \mathbf{V}^{h} \times \mathbf{U}^{h}$ is any given approximation of $(\sigma, u)$. Subtracting the equations (3.14), (3.15) from (3.3), (3.4), respectively, and recalling that $\left.\left(u-u_{c}\right)\right|_{\partial \Omega_{i}}=0$, we have

$$
\begin{aligned}
& \alpha \mathbf{a}\left(\Pi_{1} \sigma-\sigma_{h}, \tau\right)-\alpha \mathbf{b}_{2}\left(\tau, \Pi_{0} u-u_{h}\right)+\mathbf{b}_{1}\left(\tau, \Pi_{0} u-u_{h}-T_{c}\left(\Pi_{0} u-u_{h}\right)\right) \\
& \quad=\alpha \mathbf{a}\left(\Pi_{1} \sigma-\sigma, \tau\right)-\alpha \mathbf{b}_{2}\left(\tau, \Pi_{0} u-u\right)+\mathbf{b}_{1}\left(\tau, \Pi_{0} u-T_{c}\left(\Pi_{0} u\right)\right) \quad \forall \tau \in \mathbf{V}^{h},
\end{aligned}
$$

$$
\begin{aligned}
& \alpha \mathbf{b}_{2}\left(\Pi_{1} \sigma-\sigma_{h}, v\right)-\mathbf{b}_{1}\left(\Pi_{1} \sigma-\sigma_{h}, v-T_{c}(v)\right)+(1-\alpha) \mathbf{d}\left(\Pi_{0} u-u_{h}, v\right) \\
& \quad=\alpha \mathbf{b}_{2}\left(\Pi_{1} \sigma-\sigma, v\right)-\mathbf{b}_{1}\left(\Pi_{1} \sigma-\sigma, v-T_{c}(v)\right)+(1-\alpha) \mathbf{d}\left(\Pi_{0} u-u, v\right) \\
& \forall v \in \mathbf{U}^{h} .
\end{aligned}
$$

Setting up $\tau=\delta \sigma_{h}:=\Pi_{1} \sigma-\sigma_{h}$ and $v=\delta u_{h}:=\Pi_{0} u-u_{h}$ in the above equations and then adding both, we get

$$
\begin{aligned}
\alpha \mathbf{a}\left(\delta \sigma_{h},\right. & \left.\delta \sigma_{h}\right)+(1-\alpha) \mathbf{d}\left(\delta u_{h}, \delta u_{h}\right) \\
= & \left\{\alpha \mathbf{a}\left(\Pi_{1} \sigma-\sigma, \delta \sigma_{h}\right)-\alpha \mathbf{b}_{2}\left(\delta \sigma_{h}, \Pi_{0} u-u\right)+\alpha \mathbf{b}_{2}\left(\Pi_{1} \sigma-\sigma, \delta u_{h}\right)\right\} \\
& +\left\{-\mathbf{b}_{1}\left(\Pi_{1} \sigma-\sigma, \delta u_{h}-T_{c}\left(\delta u_{h}\right)\right)+(1-\alpha) \mathbf{d}\left(\Pi_{0} u-u, \delta u_{h}\right)\right\} \\
& +\mathbf{b}_{1}\left(\delta \sigma_{h}, \Pi_{0} u-T_{c}\left(\Pi_{0} u\right)\right) \\
= & \sum(\sigma, u)+\mathbf{b}_{1}\left(\delta \sigma_{h}, \Pi_{0} u-T_{c}\left(\Pi_{0} u\right)\right) .
\end{aligned}
$$


For the algebraic sum $\sum(\sigma, u)$, by virtue of the Schwarz inequality and $\left|\mathbf{b}_{2}(\tau, v)\right| \leq$ $\mathbf{a}^{\frac{1}{2}}(\tau, \tau) \cdot \mathbf{d}^{\frac{1}{2}}(v, v)$, we have

$$
\begin{aligned}
\left|\sum(\sigma, u)\right| \leq & \alpha \mathbf{a}^{\frac{1}{2}}\left(\Pi_{1} \sigma-\sigma, \Pi_{1} \sigma-\sigma\right) \cdot \mathbf{a}^{\frac{1}{2}}\left(\delta \sigma_{h}, \delta \sigma_{h}\right) \\
& +(1-\alpha) \mathbf{d}^{\frac{1}{2}}\left(\Pi_{0} u-u, \Pi_{0} u-u\right) \cdot \mathbf{d}^{\frac{1}{2}}\left(\delta u_{h}, \delta u_{h}\right) \\
& +\mid \alpha \mathbf{b}_{2}\left(\delta \sigma_{h}, \Pi_{0} u-u\right)-\alpha \mathbf{b}_{2}\left(\Pi_{1} \sigma-\sigma, \delta u_{h}\right) \\
& +\mathbf{b}_{1}\left(\Pi_{1} \sigma-\sigma, \delta u_{h}-T_{c}\left(\delta u_{h}\right)\right) \mid \\
\leq & \alpha \mathbf{a}^{\frac{1}{2}}\left(\delta \sigma_{h}, \delta \sigma_{h}\right)\left[\mathbf{a}^{\frac{1}{2}}\left(\Pi_{1} \sigma-\sigma, \Pi_{1} \sigma-\sigma\right)+\mathbf{d}^{\frac{1}{2}}\left(\Pi_{0} u-u, \Pi_{0} u-u\right)\right] \\
& +(1-\alpha) \mathbf{d}^{\frac{1}{2}}\left(\delta u_{h}, \delta u_{h}\right)\left[\mathbf{d}^{\frac{1}{2}}\left(\Pi_{0} u-u, \Pi_{0} u-u\right)\right. \\
& \left.\quad+\frac{\alpha}{1-\alpha} \mathbf{a}^{\frac{1}{2}}\left(\Pi_{1} \sigma-\sigma, \Pi_{1} \sigma-\sigma\right)\right] \\
& +C\left\|\Pi_{1} \sigma-\sigma\right\|_{\mathbf{V}}\left\|\delta u_{h}\right\|_{\mathbf{U}} .
\end{aligned}
$$

Noticing that

$$
\|v\|_{\mathbf{U}} \leq C(1-2 \nu)^{\frac{1}{2}} \mathbf{d}^{\frac{1}{2}}(v, v) \leq C_{0}\|v\|_{\mathbf{U}}
$$

and

$$
\mathbf{a}^{\frac{1}{2}}(\tau, \tau) \leq C(1-2 \nu)^{\frac{1}{2}}\|\tau\|_{\mathbf{v}}
$$

where $C$ and $C_{0}$ are independent of $\nu$ and $h$, by using the Schwarz inequality again, the above estimate yields that

$$
\begin{gathered}
\left|\sum(\sigma, u)\right| \leq\left[\alpha \mathbf{a}\left(\delta \sigma_{h}, \delta \sigma_{h}\right)+(1-\alpha) \mathbf{d}\left(\delta u_{h}, \delta u_{h}\right)\right]^{\frac{1}{2}} \\
\cdot\left[\left(\alpha+\frac{\alpha^{2}}{1-\alpha}\right) \mathbf{a}\left(\Pi_{1} \sigma-\sigma, \Pi_{1} \sigma-\sigma\right)\right. \\
\left.\quad+(\alpha+1-\alpha) \mathbf{d}\left(\Pi_{0} u-u, \Pi_{0} u-u\right)+\frac{C(1-2 \nu)}{1-\alpha}\left\|\Pi_{1} \sigma-\sigma\right\|_{\mathbf{V}}^{2}\right]^{\frac{1}{2}} \\
\leq C\left[\alpha \mathbf{a}\left(\delta \sigma_{h}, \delta \sigma_{h}\right)+(1-\alpha) \mathbf{d}\left(\delta u_{h}, \delta u_{h}\right)\right]^{\frac{1}{2}} \\
\cdot\left[\left(\frac{1-2 \nu}{1-\alpha}\right)\left\|\Pi_{1} \sigma-\sigma\right\|_{\mathbf{V}}^{2}+\frac{1}{1-2 \nu}\left\|u-\Pi_{0} u\right\|_{\mathbf{U}}^{2}\right]^{\frac{1}{2}} .
\end{gathered}
$$

From this estimate and (3.18), we can deduce that

$$
\begin{aligned}
& {\left[\alpha \mathbf{a}\left(\delta \sigma_{h}, \delta \sigma_{h}\right)+(1-\alpha) \mathbf{d}\left(\delta u_{h}, \delta u_{h}\right)\right]^{\frac{1}{2}}} \\
& \leq C\left\{\left[\left(\frac{1-2 \nu}{1-\alpha}\right)\left\|\sigma-\Pi_{1} \sigma\right\|_{\mathbf{V}}^{2}+\frac{1}{1-2 \nu}\left\|u-\Pi_{0} u\right\|_{\mathbf{U}}^{2}\right]^{\frac{1}{2}}\right. \\
& \left.\quad+[\alpha(1-2 \nu)]^{-\frac{1}{2}} \sup _{\delta \sigma_{h}} \frac{\mathbf{b}_{1}\left(\delta \sigma_{h}, \Pi_{0} u-T_{c}\left(\Pi_{0} u\right)\right)}{\left\|\delta \sigma_{h}\right\|_{\mathbf{V}}}\right\} .
\end{aligned}
$$


Thus, by triangular inequality, we obtain

$$
\begin{aligned}
& {\left[\alpha(1-2 \nu)\left\|\sigma-\sigma_{h}\right\|_{0, \Omega}^{2}+\left(\frac{1-\alpha}{1-2 \nu}\right)\left\|u-u_{h}\right\|_{\mathbf{U}}^{2}\right]^{\frac{1}{2}}} \\
& \left.\quad \leq \quad \alpha \mathbf{a}\left(\sigma-\Pi_{1} \sigma, \sigma-\Pi_{1} \sigma\right)+(1-\alpha) \mathbf{d}\left(u-\Pi_{0} u, u-\Pi_{0} u\right)\right]^{\frac{1}{2}} \\
& \quad+\left[\alpha \mathbf{a}\left(\delta \sigma_{h}, \delta \sigma_{h}\right)+(1-\alpha) \mathbf{d}\left(\delta u_{h}, \delta u_{h}\right)\right]^{\frac{1}{2}} \\
& \leq C\left\{\left(\frac{1-2 \nu}{1-\alpha}\right)^{\frac{1}{2}}\left\|\sigma-\Pi_{1} \sigma\right\|_{\mathbf{V}}+\left(\frac{1}{1-2 \nu}\right)^{\frac{1}{2}}\left\|u-\Pi_{0} u\right\|_{\mathbf{U}}\right. \\
& \left.\quad+[\alpha(1-2 \nu)]^{-\frac{1}{2}} \sup _{\tau \in \mathbf{V}^{h}} \frac{\mathbf{b}_{1}\left(\tau, \Pi_{0} u-T_{c}\left(\Pi_{0} u\right)\right)}{\|\tau\|_{\mathbf{V}}}\right\} .
\end{aligned}
$$

Because this estimate holds for any $\left(\Pi_{1} \sigma, \Pi_{0} u\right) \in \mathbf{V}^{h} \times \mathbf{U}^{h}$, the theorem is proved.

For helping various applications of the theorem, we want to show that a lot of nonconforming finite element spaces can be viewed as the so-called strain-enriched subspace. Particularly, we have the following proposition.

Proposition 3.1. If a continuity node point set $S_{i}$ of a nonconforming space $\mathbf{U}^{h}$ contains all the vertices of $\Omega_{i}, \mathbf{U}^{h}$ can be regarded as a strain-enriched subspace, $T_{c}(v)=v_{c}$ is the conforming part of $v$, and

$$
\left\|v-T_{c}(v)\right\|_{\frac{1}{2}, \partial \Omega_{i}} \leq C\|\varepsilon(v)\|_{0, \Omega_{i}} \quad \text { and } \quad\left\|v-T_{c}(v)\right\|_{\frac{1}{2}, \partial \Omega_{i}} \leq C h_{i}|v|_{2, \Omega_{i}},
$$

where $C$ is independent of $h_{i}$.

Proof. By virtue of the continuity of $v \in \mathbf{U}^{h}$ at the vertices, it is known from [2] that there exists a unique piecewise linear (or bilinear) interpolant $v_{L} \in C^{(0)}(\Omega)$ such that for any vertex $q$ of $\Omega_{i}, v-v_{\left.L\right|_{q}}=0$, and

$$
\left|v-v_{L}\right|_{t, \Omega_{i}} \leq C h_{i}^{2-t}|v|_{2, \Omega_{i}}, t=0,1
$$

where $C$ is independent of $h_{i}$.

Since $v_{c}=T_{c}(v)$ or $v_{L}$ is a conforming component of $T_{C}(v)$, the estimates (3.19) imply that

$$
\left\|v-T_{c}(v)\right\|_{\frac{1}{2}, \partial \Omega_{i}} \leq C\left|v-T_{c}(v)\right|_{1, \Omega_{i}} \leq C h_{i}|v|_{2, \Omega_{i}}
$$

that is, the second inequality in the proposition is proved.

Now we turn to prove the first inequality. By the uniqueness of the Lagrange interpolation $T_{c}(v)=v_{L}$ we can conclude that for $v \in \mathbf{U}^{h} \cap\left(H_{0}^{1}(\Omega)\right)^{n}, T_{c}(v)=v$ and $\|\varepsilon(v)\|_{0, \Omega_{i}}=0$ leads to $v-T_{c}(v)=0$. Thus, by compactness of a bounded sequence in the finite dimensional space $\mathbf{U}^{h}$ and by reduction to absurdity, we can deduce that there exists a constant $C\left(\Omega_{i}\right)>0$, such that for $v \in \mathbf{U}^{h}\left(\Omega_{i}\right)$,

$$
\left|v-T_{c}(v)\right|_{1, \Omega_{i}} \leq C\left(\Omega_{i}\right)\|\varepsilon(v)\|_{0, \Omega_{i}} .
$$

By a scaling argument (for details, see the similar discussion in [16] or [17]), we can confirm that

$$
\sup _{\substack{\Omega_{i} \in \mathbb{T}_{h} \\ 0<h_{i} \leq 1}} \sup _{v \in \mathbf{U}^{h}} \frac{\left|v-T_{c}(v)\right|_{1, \Omega_{i}}}{\|\varepsilon(v)\|_{0, \Omega_{i}}}=C<\infty ;
$$

that is, $C\left(\Omega_{i}\right)$ in (3.21) can be independent of $h_{i}$. Then it follows from (3.20), (3.21) that the first inequality is proved. 
Remark 3.1. It is obvious that Wilson's $n$-quadrilateral $\mathbf{U}_{W}^{h}$ is an example that the assumption of Proposition 3.1] can be fulfilled. By virtue of (3.20), Theorem 3.2 concludes that, for the combination of $\mathbf{U}_{W}^{h}$ with $\mathbf{V}_{r}^{h}$ and $\alpha \neq 0,1$, the corresponding combined hybrid methods $C H(r)$ are convergent by rate $O(h)$ as $h \rightarrow 0$. But, if $\alpha=1, C H(0)$ becomes the conventional assumed stress hybrid method and does not converge due to rank deficiency of element stiffness matrices.

Remark 3.2. Due to the feature that the stress variables can be eliminated at the element level, the combined hybrid method can be considered as a generalized incompatible displacement scheme. Theorem 3.2 shows that $\alpha=\frac{1}{2}$ is, in general, a rational and safe choice.

\section{ACCURACY-Enhanced CONDItION: EnERGy COMPATIBILITy}

In this section we discuss under what condition the enhancement of accuracy and stability, mentioned in the introduction, can be achieved by the combined hybrid scheme.

Due to appearance of the error term $\sup _{\tau} \frac{\mathbf{b}_{1}\left(\tau, v-T_{c}(v)\right)}{\|\tau\|_{\mathbf{v}}}$ in the error estimate of Theorem 3.2, the optimal error estimate that is of the same order as

$$
\left(\inf _{\tau \in \mathbf{V}^{h}}\|\sigma-\tau\|_{\mathbf{V}}+\inf _{v \in \mathbf{U}^{h}}\|u-v\|_{\mathbf{U}}\right)
$$

cannot in general be obtained. Therefore, for the construction of a "good" scheme, it is of crucial importance to reduce the error caused by $\mathbf{b}_{1}\left(\tau, v-T_{c}(v)\right)$. As far as a strain-enhanced space $\mathbf{U}^{h}$ that $\inf _{v \in \mathbf{U}^{h}}\|u-v\|_{\mathbf{U}}$ is smaller than $\inf _{v \in \mathbf{U}^{h}}\left(\sum_{i} \| u-\right.$ $\left.T_{c}(v) \|_{\frac{1}{2}, \partial \Omega_{i}}^{2}\right)^{\frac{1}{2}}$, an approach to the reduction is to construct $\mathbf{V}^{h}$ appropriately so that $\oint_{\partial \Omega_{i}} \tau \cdot \vec{n} \cdot\left(v-T_{c}(v)\right) d s$ is small enough.

Definition 4.1. A strain-enriched finite element space $\mathbf{U}^{h}$ is referred to as $(l, k)$ order energy-compatible (or $E$-compatible) with respect to the stress space $\mathbf{V}^{h}$ if there exist two constants $l, k>0$ and $C>0$, independent of $h_{i}$, such that for any $\Omega_{i} \in \mathrm{T}_{h}, v \in \mathbf{U}^{h}$,

1) $\left\|v-T_{c}(v)\right\|_{\frac{1}{2}, \partial \Omega_{i}} \leq C\|\varepsilon(v)\|_{0, \Omega_{i}}$,

2) $\left\|v-T_{c}(v)\right\|_{\frac{1}{2}, \partial \Omega_{i}} \leq C h_{i}^{l}|v|_{l+1, \Omega_{i}}$,

3) $\left|\oint_{\partial \Omega_{i}} T(\tau) \cdot\left(v-T_{c}(v)\right) d s\right| \leq C h_{i}^{k}\|\tau\|_{\mathbf{V}, \Omega_{i}} \cdot\left\|v-T_{c}(v)\right\|_{\frac{1}{2}, \partial \Omega_{i}} \quad \forall \tau \in \mathbf{V}^{h}$.

For convenience, the pair $(l, k)$ is named as the index of $E$-compatibility in what follows, and we denote the incompatible part $v-T_{C}(v)$ of $v$ by $v_{I}$.

Theorem 4.1. (I) For the combined hybrid scheme (3.14),(3.15) with index $(l, k)$ of E-compatibility, we have the error estimates of so-called accuracy-enhanced type

$$
\begin{aligned}
\left\|\sigma-\sigma_{h}\right\|_{0, \Omega}+\left(\frac{1-\alpha}{\alpha}\right)^{\frac{1}{2}}(1-2 \nu)^{-1}\left\|u-u_{h}\right\|_{\mathbf{U}} \\
\leq C[\alpha(1-\alpha)]^{-\frac{1}{2}} \cdot\left\{\inf _{\tau \in \mathbf{V}^{h}}\|\sigma-\tau\|_{\mathbf{V}}+(1-2 \nu)^{-1}\right. \\
\left.\quad \inf _{v \in \mathbf{U}^{h}}\left[\|u-v\|_{\mathbf{U}}+h^{l+k}\left(\sum_{i}|v|_{1+l, \Omega_{i}}^{2}\right)^{\frac{1}{2}}\right]\right\} .
\end{aligned}
$$


(II) In addition, assume that $u \in\left(H_{0}^{1}(\Omega) \cap H^{m+1}(\Omega)\right)^{n}, m \geq l$ and

$$
\widehat{P}_{m}\left(F_{i}^{-1}\right) \subset \mathbf{U}^{h}\left(\Omega_{i}\right) \quad \forall \Omega_{i} \in \mathrm{T}_{h} .
$$

Then the following error estimates of locking-free convergence hold uniformly for $\nu \leq 0.5-\frac{h}{2}$ as $h \rightarrow 0$ :

$$
\begin{aligned}
\| \sigma- & \sigma_{h}\left\|_{0, \Omega}+\left(\frac{1-\alpha}{\alpha}\right)^{\frac{1}{2}}(1-2 \nu)^{-1}\right\| u-u_{h} \|_{\mathbf{U}} \\
& \leq C[\alpha(1-\alpha)]^{-\frac{1}{2}} \cdot\left\{\inf _{\tau \in \mathbf{V}^{h}}\|\sigma-\tau\|_{\mathbf{V}}+\left(h^{m-1}+h^{l+k-1}\right)\|u\|_{l+1, \Omega}\right\} .
\end{aligned}
$$

Proof. Because the index $(l, k)$ of $E$-compatibility implies that

$$
\begin{aligned}
\left|\mathbf{b}_{1}\left(\tau, v_{I}\right)\right| & \leq \sum_{i}\left|\oint_{\partial \Omega_{i}} \tau \cdot \vec{n} \cdot v_{I} d s\right| \leq C \sum_{i}\left(h_{i}^{k}\|\tau\|_{\mathbf{V}, \Omega_{i}} \cdot\left\|v-T_{c}(v)\right\|_{\frac{1}{2}, \partial \Omega_{i}}\right) \\
& \leq C h^{k+l} \sum_{i}\left(\|\tau\|_{\mathbf{V}, \Omega_{i}} \cdot|v|_{1+l, \Omega_{i}}\right) \leq C h^{k+l}\|\tau\|_{\mathbf{V}}\left(\sum_{i}|v|_{1+l, \Omega_{i}}^{2}\right)^{\frac{1}{2}},
\end{aligned}
$$

the first conclusion of Theorem 4.1 can be derived immediately from Theorem 3.2

Next, if the hypothesis in (II) is satisfied as well, it is well known (see [2]) that there exist $\Pi_{0} u \in \mathbf{U}^{h}$ and a constant $C>0$, independent of $h_{i}$ and $u$, such that

$$
\left\|u-\Pi_{0}(u)\right\|_{t, \Omega_{i}} \leq C h^{m+1-t}\|u\|_{m+1, \Omega}, \quad t=0,1
$$

and

$$
\left|\Pi_{0}(u)\right|_{l+1, \Omega_{i}} \leq C\|u\|_{l+1, \Omega_{i}} .
$$

Thus, in view of the assumption $h \leq 1-2 \nu$, we get

$$
\begin{aligned}
& (1-2 \nu)^{-1} \inf _{v \in \mathbf{U}^{h}}\left[\|u-v\|_{\mathbf{U}}+h^{l+k}\left(\sum_{i}|v|_{1+l, \Omega_{i}}^{2}\right)^{\frac{1}{2}}\right] \\
& \leq(1-2 \nu)^{-1}\left[\left\|u-\Pi_{0}(u)\right\|_{\mathbf{U}}+h^{l+k}\left(\sum_{i}\left|\Pi_{0}(u)\right|_{1+l, \Omega_{i}}^{2}\right)^{\frac{1}{2}}\right] \\
& \quad \leq C\left[h^{m-1}+h^{l+k-1}\right] \cdot\|u\|_{m+1, \Omega},
\end{aligned}
$$

which means that the proof has been finished.

Now we turn to discuss how to identify the index of $E$-compatibility for a given incompatible space as well as how to construct $\left(\mathbf{U}^{h}, \mathbf{V}^{h}\right)$ so that a higher E-C index can be achieved. In order to construct the high performance schemes, we will consider Wilson displacement space $\mathbf{U}_{W}^{h}$ in what follows.

First, following the paper [15], we introduce a condition on mesh subdivisions.

Condition (B). The distance $d_{\Omega_{i}}$ between the midpoints of the diagonals of $\Omega_{i} \in$ $\mathrm{T}_{h}$ is of order $O\left(h_{i}^{2}\right)$ uniformly for all element $\Omega_{i}$ as $h_{i} \rightarrow 0$.

It has already been mentioned in the paper [15] that the quadrilaterals, produced by bisection scheme of mesh subdivisions, satisfy Condition (B). 
Proposition 4.1. Assume that Condition (B) is fulfilled. Then the index $(l, k)$ of E-compatibility of Wilson's quadrilateral with respect to the stress space $\mathbf{V}_{0}^{h}=$ $\left\{\tau \in \mathbf{V}|: \tau|_{\Omega_{i}}=\right.$ const $\}$ is equal to $(1,1)$.

Proof. By Green's formula $\oint_{\partial \Omega_{i}} \tau \cdot \vec{n} \cdot v_{I} d s=\int_{\Omega_{i}}\left[\operatorname{div} \tau \cdot v_{I}+\tau \cdot \varepsilon\left(v_{I}\right)\right] d \Omega$, for $(\tau, v) \in \mathbf{V}_{0}^{h} \times \mathbf{U}_{W}^{h}$, we get $\left|\oint_{\partial \Omega_{i}} \tau \cdot \vec{n} \cdot v_{I} d s\right| \leq\left|\tau \cdot \int_{\Omega_{i}} \varepsilon\left(v_{I}\right) d \Omega\right|$.

Recalling that $\hat{v}_{I}=\overline{\left(v-T_{c}(v)\right)}=\sum_{t=1}^{2}\left[v_{I, t}\left(1-\xi_{t}^{2}\right)\right] v_{I, t} \in R^{2}$ and $v_{I}=\hat{v}_{I}\left(F_{i}^{-1}\right)$, where $F_{i}=\sum_{i=1}^{4}\left(1+\xi_{1, t} \xi_{1}\right)\left(1+\xi_{2, t} \xi_{2}\right) x_{i, t}$ denotes the element mapping from the vertex $\left(\xi_{1, t}, \xi_{2, t}\right)$ of square $\widehat{K}=[-1,1] \times[-1,1]$ to the vertex $x_{i, t}$ of $\Omega_{i}$, and its Jacobian is defined by

$$
[J]=\left[\begin{array}{ll}
\frac{\partial x^{(1)}}{\partial \xi_{1}} & \frac{\partial x^{(2)}}{\partial \xi_{1}} \\
\frac{\partial x^{(1)}}{\partial \xi_{2}} & \frac{\partial x^{(2)}}{\partial \xi_{2}}
\end{array}\right]=\left[\begin{array}{ll}
a_{1}+a_{2} \xi_{2} & b_{1}+b_{2} \xi_{2} \\
a_{3}+a_{2} \xi_{1} & b_{3}+b_{2} \xi_{1}
\end{array}\right],
$$

$a_{t}$ and $b_{t}(t=1,2,3)$ are some algebraic sums of the coordinates of the vertex $x_{i, t}$. Particularly

$$
\left\{\begin{array}{l}
a_{2}=\frac{1}{4}\left(x_{i, 1}^{(1)}-x_{i, 2}^{(1)}+x_{i, 3}^{(1)}-x_{i, 4}^{(1)}\right), \\
b_{2}=\frac{1}{4}\left(x_{i, 1}^{(2)}-x_{i, 2}^{(2)}+x_{i, 3}^{(2)}-x_{i, 4}^{(2)}\right)
\end{array}\right.
$$

as shown in [14], $\max \left(\left|a_{2}\right|,\left|b_{2}\right|\right) \leq C d_{\Omega_{i}} \leq C h_{i}^{2}$.

By some simple computations (see [14]), we can obtain

$$
\begin{aligned}
& \left|\oint_{\partial \Omega_{i}} \tau \cdot \vec{n} \cdot v_{I} d s\right| \leq\left|\tau \cdot \int_{\Omega_{i}} \varepsilon\left(v_{I}\right) d \Omega\right| \\
& \quad \leq C\left[\frac{\max \left(\left|a_{2}\right|,\left|b_{2}\right|\right)}{h_{i}}\right]\|\tau\|_{0, \Omega_{i}}\left(\left|v_{I, 1}\right|+\left|v_{I, 2}\right|\right) \\
& \quad \leq C h_{i}\|\tau\|_{0, \Omega_{i}}\left\|\varepsilon\left(v_{I}\right)\right\|_{0, \Omega_{i}} .
\end{aligned}
$$

Then this estimation and Proposition 3.1 together conclude that Proposition 4.1 is true.

Proposition 4.2. Assume that Condition (B) is fulfilled. Then the index $(l, k)$ of E-compatibility of Wilson's quadrilateral with respect to Pian-Sumihara's stress space (proposed in the paper [9] and denoted here by $\mathbf{V}_{P S}^{h}$ ) is equal to $(1,1)$.

Proof. Notice that for $\tau \in \mathbf{V}_{P S}^{h}$, as defined in [9],

$$
\left.\tau\right|_{\Omega_{i}}=\left[\begin{array}{ccccc}
1 & 0 & 0 & a_{1}^{2} \xi_{2} & a_{3}^{2} \xi_{1} \\
0 & 1 & 0 & b_{1}^{2} \xi_{2} & b_{3}^{2} \xi_{1} \\
0 & 0 & 1 & a_{1} b_{1} \xi_{2} & a_{3} b_{3} \xi_{1}
\end{array}\right]\left[\begin{array}{c}
\beta_{1} \\
\vdots \\
\beta_{5}
\end{array}\right] .
$$

Let us divide $\tau \in \mathbf{V}_{P S}^{h}$ into $\tau_{1}$ and $\tau_{2}, \tau=\tau_{1}+\tau_{2}, \tau_{1} \in \mathbf{V}_{0}^{h}$ is a piecewise constant stress. As shown in [10], for $\tau \in \mathbf{V}_{P S}^{h}$ and $v \in \mathbf{U}_{W}^{h}$,

$$
\oint_{\partial \Omega_{i}} \tau_{2} \cdot \vec{n} \cdot v_{I} d s=0 \quad \forall \Omega_{i} \in \mathrm{T}_{h}
$$

Thus, by virtue of Proposition 4.1,

$$
\begin{aligned}
\left|\oint_{\partial \Omega_{i}} \tau \cdot \vec{n} \cdot v_{I} d s\right| & =\left|\oint_{\partial \Omega_{i}} \tau_{1} \cdot \vec{n} \cdot v_{I} d s\right| \\
& \leq C h_{i}\left\|\tau_{1}\right\| \mathbf{V}, \Omega_{i}\left\|\varepsilon\left(v_{I}\right)\right\|_{0, \Omega_{i}} \leq C h_{i}\|\tau\|_{\mathbf{V}, \Omega_{i}}\left\|\varepsilon\left(v_{I}\right)\right\|_{0, \Omega_{i}},
\end{aligned}
$$

which confirms that the proposition is true as well. 
Let us denote two 4-node combined hybrid elements, constructed by using $\mathbf{V}_{r}^{h} \times$ $\mathbf{U}_{W}^{h}$ and $\mathbf{V}_{P S}^{h} \times \mathbf{U}_{W}^{h}$, respectively by $C H(r)$ and $C H(P S)$. By virtue of Propositions 4.1 and 4.2 the hypothesis of Theorem 4.1 is fulfilled, respectively, for $C H(0)$ element and $C H(P S)$ element. In addition, the inclusion $\widehat{P}_{2}\left(F_{i}^{-1}\right) \subseteq \mathbf{U}_{W}^{h}$ is satisfied. Theorem 4.1 states that $C H(0)$ and $C H(P S)$ can be considered as two accuracy-enhanced schemes.

Proposition 4.3. Assume that $u \in\left(H_{0}^{1}(\Omega) \cap H^{3}(\Omega)\right)^{n}$ and Condition (B) is fulfilled. For the finite element solutions $\left(\sigma_{h, 0}, u_{h, 0}\right)$ and $\left(\sigma_{h, P S}, u_{h, P S}\right)$ determined respectively by $\mathrm{CH}(0)$ and $C H(P S)$, we have

$$
\begin{aligned}
\| \sigma- & \sigma_{h, 0}\left\|_{0, \Omega}+\left(\frac{1-\alpha}{\alpha}\right)^{\frac{1}{2}}(1-2 \nu)^{-1}\right\| u-u_{h, 0} \|_{\mathbf{U}} \\
\leq & C[\alpha(1-\alpha)]^{-\frac{1}{2}} \cdot\left\{\inf _{\tau \in \mathbf{V}_{0}^{h}}\|\sigma-\tau\|_{\mathbf{V}}+\left(\frac{h^{2}}{1-2 \nu}\right)\|u\|_{3, \Omega}\right\} \\
\leq & C[\alpha(1-\alpha)]^{-\frac{1}{2}} \cdot\left\{h\|u\|_{2, \Omega}+h\|u\|_{3, \Omega}\right\}
\end{aligned}
$$

and

$$
\begin{aligned}
\| \sigma- & \sigma_{h, P S}\left\|_{0, \Omega}+\left(\frac{1-\alpha}{\alpha}\right)^{\frac{1}{2}}(1-2 \nu)^{-1}\right\| u-u_{h, P S} \|_{\mathbf{U}} \\
& \leq C[\alpha(1-\alpha)]^{-\frac{1}{2}} \cdot\left\{\inf _{\tau \in \mathbf{V}_{P S}^{h}}\|\sigma-\tau\|_{\mathbf{v}}+\left(\frac{h^{2}}{1-2 \nu}\right)\|u\|_{3, \Omega}\right\} \\
& \leq C[\alpha(1-\alpha)]^{-\frac{1}{2}} \cdot\left\{h\|u\|_{2, \Omega}+h\|u\|_{3, \Omega}\right\},
\end{aligned}
$$

where $C$ is independent of $h$ and $\nu, \nu \leq 0.5-\frac{h}{2}$ as $h \rightarrow 0$.

Remark 4.1. Notice that $\inf _{\tau \in \mathbf{V}_{P S}^{h}}\|\sigma-\tau\|_{\mathbf{V}}<\inf _{\tau \in \mathbf{V}_{0}^{h}}\|\sigma-\tau\|_{\mathbf{V}}$, we can believe that the degree of accuracy of the $C H(P S)$ element is higher than that of the $C H(0)$ element. Furthermore, the numerical experiments [19] show that $C H(0)$ and $C H(P S)$ are locking free, and $C H(P S)$ is much more accurate than the classical conforming bilinear element when using coarse meshes. Therefore, Proposition 4.3 provides a theoretical interpretation for why numerical results of 4-node enriched stress/strain elements, such as $C H(P S)$, are of high performance. For the details on numerical experiments, see the published paper [19].

Remark 4.2. Numerical experiments in [19] also show that, by contrast with $C H(0)$ and $C H(P S)$, the numerical performance of another combined hybrid element $C H(1)$, constructed by $\mathbf{V}_{1}^{h}$ and $\mathbf{U}_{W}^{h}$, is quite poor due to $E$-compatibility deficiency. Therefore whether $\mathbf{b}_{1}(\tau, v-T(v)) \approx 0$ or not is a key point for the construction of "good" hybrid elements.

\section{ACKNOWLEDGMENTS}

I would like to thank the referees for their valuable suggestions, which helped improve this paper. 


\section{REFERENCES}

1. Brezzi F., Fortin, M: Mixed and hybrid finite element methods. Springer-Verlag, 1991. MR 92d:65187

2. Ciarlet P. G.: The Finite Element Method for Elliptic Problems: North-Holland, Amsterdam, 1978. MR 58:25001

3. Bochev P. B., Gunzburger M. D.: Finite element methods of least-squares type, SIAM Review 40 (1998), 789-837. MR 99k:65104

4. Franca L. P., Highes T. J. R.: Two classes of mixed finite element methods. Comput. Methods Appl. Mech. Engrg. 69 (1988), 89-129. MR 90b:65202

5. Hughes T. J. R., France L. P., Balestra M.: A new finite element formulation of computational fluid dynamics, a stable Petrov-Galerkin formulation of the stokes problem accommodating equal-order interpolations. Comput. Methods Appl. Mech. Engrg. 59 (1986), 85-99. MR 89j:76015d MR 89j:76015e

6. Kang D. S., Pian T. H. H.: A 20-DOF hybrid general shell element. Comput. Struct. 30 (1988), 789-794.

7. Mikhlin S. G.: The problem of the minimum of a quadratic functional. Holden-Day, San Francisco, London, Amsterdam, 1965. MR 30:1427

8. Pehlivanov A. I., Carey G. F., Vassilevski P. S.: Least-squares mixed finite element methods for non-selfadjoint elliptic problem: I. Error estimates. Numer. Math. 72 (1996), 501-522. MR 97f:65068

9. Pian T. H. H., Sumihara K.: Rational approach for assumed stress finite elements. Inter. J. Numer. Meth. Engrg. 20 (1984), 1685-1695.

10. Pian T. H. H., Wu C.-C.: A rational approach for choosing stress terms for hybrid finite element formulations. Internat. J. Numer. Meth. Engrg. 26 (1988), 2331-2343. MR 89f:73052

11. Piltner P., Taylor, R. L.: A quadrilateral mixed finite element with two enhanced strain modes. Internat. J. Numer. Meth. Engrg. 38 (1995), 1783-1808. MR 96a:73062

12. Piltner R., Taylor R. L.: A systematic construction of $\mathbf{B}$-bar functions for linear and nonlinear mixed-enhanced finite elements for plane elasticity problems. Internat. J. Numer. Meth. Engrg. 44 (1999), 615-639. MR 99j:73099

13. Reddy B. D., Simo J. C.: Stability and convergence of a class of enhanced strain methods, SIAM J. Numer. Anal. 32 (1995), 1705-1728. MR 96k:73082a

14. Simo J. C., Rifai M. S.: A class of mixed assumed strain methods and the method of incompatible modes. Internat. J. Numer. Meth. Engrg. 29 (1990), 1595-1638. MR 91d:73062

15. Shi. Z.-C.: A convergence condition for the quadrilateral Wilson element. Numer. Math. 44 (1984), 349-361. MR 86d:65151

16. Zhou T.-X.: Equivalency theorem for "saddle point" finite element schemes and two criteria of strong Babuška-Brezzi condition. Sci. Sinica 24 (1981), 1190-1206. MR 83e:65182

17. Zhou T.-X.: Mixed stiffness finite element (I) (II), Sci. Sinica Ser. A 29 (1986), 1233-1251; 30 (1987), 1-11. MR 88h:65221 MR 88h:65222

18. Zhou T.-X.: Finite element method based on combination of "saddle point" variational formulations. Sci. China Ser. E 40 (1997), 285-300. MR 98e:65103

19. Zhou T.-X., Nie Y.-F.: Combined hybrid approach to finite element schemes of high performance. Internat. J. Numer. Meth. Engrg. 50 (2001), 181-202. MR 2002a:74125

Aeronautical Computing Technology Research Institute, Xi'an 710068, Peoples RePUBLIC OF CHINA

E-mail address: txzhou@163.net 